This item was submitted to Loughborough's Research Repository by the author.

Items in Figshare are protected by copyright, with all rights reserved, unless otherwise indicated.

\title{
Effects of contaminant decay on the diffusion centre of a river
}

PLEASE CITE THE PUBLISHED VERSION

LICENCE

CC BY-NC-ND 4.0

\section{REPOSITORY RECORD}

Mebine, P., and Ron Smith. 2019. "Effects of Contaminant Decay on the Diffusion Centre of a River". figshare. https://hdl.handle.net/2134/248. 


\title{
Effects of Contaminant Decay on the Diffusion Centre of a River
}

\author{
P. Mebine* and R. Smith ${ }^{\dagger}$ \\ Department of Mathematical Sciences, Loughborough University, \\ LE11 3TU, United Kingdom
}

\begin{abstract}
Many contaminants exhibit decay (radioactive decay, consumed by bacteria, heat loss or evaporation through the surface, dissolution by turbulence). For a non-symmetric river with non-reversing flow, the effects of decay are allowed for in specifying the diffusion centre i.e. the optimal postion for a steady discharge. Three families of exact solutions are presented that illustrate the effect on the diffusion centre of cross-channel variation in the decay (uniform, decreasing or increasing with depth). The diffusion centre is shifted to deeper or to shallower water accordingly as the temporal decay divided by flow speed decreases or increases with water depth.

Keywords: contaminant decay, diffusion centre, environmental impact, pollutant modes, variability of decay
\end{abstract}

\section{Introduction}

Modern large-scale sewage works and industrial processes are designed to avoid intermittent high-level waste-water discharges, and instead are aimed at steady low-level discharges. The environmental impact can be further reduced by careful selection of the discharge location. In rivers, the near-shore or littoral zones contain more abundant plant, fish and animal communities than the main channel. A commonly advocated managerial policy to conserve these areas of economic importance is to choose the source location of large-scale contaminant discharges to avoid excessive shoreline concentrations. The magnitude of avoidable shoreline excesses is shown in figure 1 for the illustrative case of a conserved solute discharged at a steady rate from a single point into a channel with water depth that increases from zero at the left to maximum water depth at the right of figure 1 .

${ }^{*}$ Corresponding Author, E-mail: P. Mebine@lboro.ac.uk

${ }^{\dagger}$ Prof. of Mathematics 


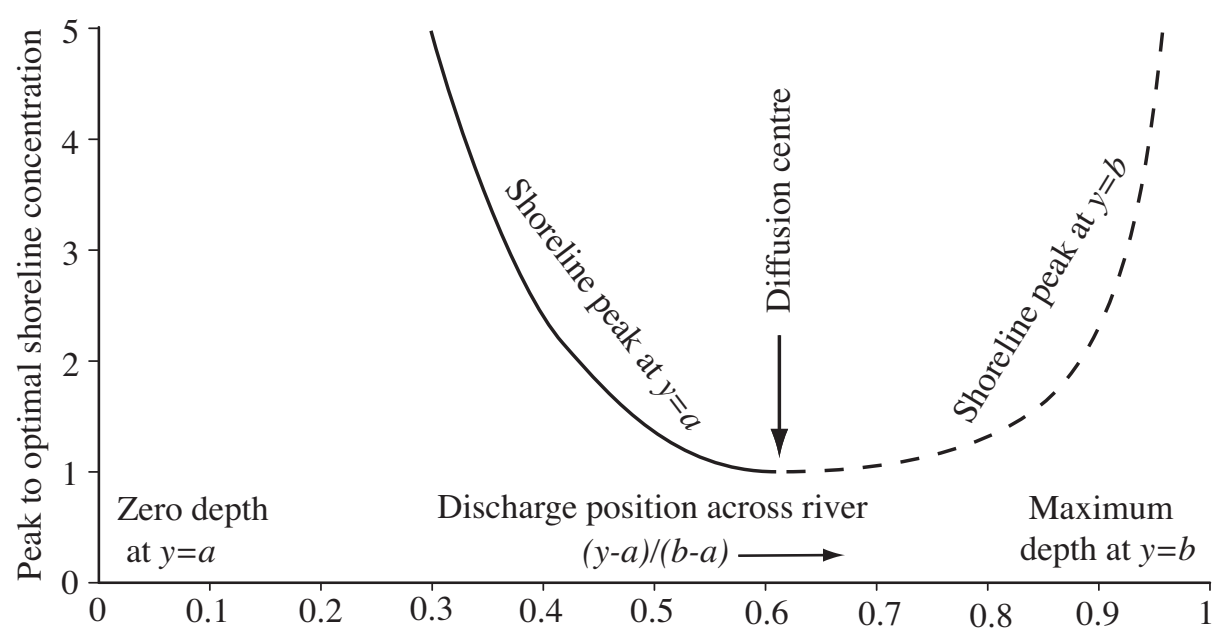

Figure 1: Magnitude of shoreline pollution if a discharge is not at the diffusion centre.

Yotsukura and Cobb [19] used the degree of mixing as a measure of the effectiveness of different discharges of non-decaying solutes in a river. Their criterion for the optimal site, or diffusion centre, is that the transverse mixing distance be minimized with complete mixing achieved as quickly as possible. For a laterally symmetric river the diffusion centre coincides with the geometric centre. In general, the diffusion centre tends to be displaced towards the deeper side of a river (as in figure 1) and close to the centre with respect to volume discharge [17]. A nice consequence of positioning a steady discharge at the diffusion centre is that at both banks the concentrations gradually increase downstream without local pollution hot-spots along either shore [15]. In figure 1, the peak shoreline pollution increases quadratically for discharge sites away from the diffusion centre, reaching 0.4 above optimal for displacements of an eighth of a channel breadth away from the diffusion centre.

Many contaminants exhibit decay. Decay mechanisms include consumption by bacteria or radioactive decay (temporal decay uniform across the flow), heat loss or evaporation through the surface (decay decreasing with depth), and break up by turbulence (decay proportional to the product of velocity and depth). For slow-moving rivers with widths of a few hundreds of metres, the time scales for transverse mixing can be of order a day and comparable with the time scales for decay. So, decay cannot be regarded as a minor perturbation that simply lowers the concentration. There are numerical models in literature which allow for the decay of contaminants $[1,9,18]$. However, no previous investigation of the effects of decay on the discharge sites has been attempted.

In the present paper a mathematical model is used to include a profile of decay in a definition of the diffusion centre for a non-symmetric straight river with non-reversing flow. Three families of exact solutions are given with profiles of decay corresponding to consumption by bacteria, to evaporation and to break up by turbulence. It is found that the diffusion centre shifts to deeper or shallower water accordingly as the the temporal decay divided by flow speed (local downstream spatial decay rate) decreases or increases with water depth. 


\section{Governing equations}

An account of flow models of river mixing by Rutherford [13] suggested that transverse mixing is more important in water quality management than either vertical or longitudinal mixing, especially when dealing with discharge of water from point sources or the mixing of tributary inflows. In natural streams it is known that the width to depth aspect ratio is large and therefore vertical equilibrium (usually vertically uniform) is achieved very much more rapidly than transverse equilibrium. Thus, for lateral mixing of solute from a steady discharge in nonstratified unidirectional flow, the concentration can be regarded as being in vertical equilibrium, and hence attention is focused along and across the flow. Steady contaminant plumes also have a large length to width ratio, which makes the effect of transverse turbulent diffusion more important than longitudinal shear dispersion [3].

For a straight channel with a cross-section that is unchanging in the downstream direction, the steady-state vertical-equilibrium advection-diffusion equation, incorporating a first-order decay parameter $\lambda(y)$ that is a function of the transverse coordinate $y$ only, is

$$
\lambda h c+h u \frac{\partial c}{\partial x}-\frac{\partial}{\partial y}\left(h \kappa \frac{\partial c}{\partial y}\right)=0
$$

The no-flux boundary conditions at the shorelines are

$$
h \kappa \frac{\partial c}{\partial y}=0 \text { on } y=a, b .
$$

Here $x$ and $y$ are the longitudinal and transverse coordinates, $c(x, y)$ is the contaminant concentration between the shorelines $y=a, b$ in water of depth $h(y) \geq 0$, steady nonreversing velocity $u(y) \geq 0$, and transverse diffusivity $\kappa(y) \geq 0$. It is not necessary that $\lambda(y)$ be single signed, but all the examples in this paper concern decaying rather than growing contaminants. If the vertical equilibrium is non-uniform (light oils, heavy oils, bed sorbtion, death of bacteria in bright sunlight near the surface), then $c, u$ and $\kappa$ are appropriately weighted vertical averages [16].

\section{Pollutant modes}

To solve the system $(1,2)$, the method of modes is employed. The eigenmodes are herein described as the pollutant modes. A separation of variables solution:

$$
c(x, y)=\sum_{n=0}^{\infty} C_{n} \exp \left(-\mu_{n} x\right) \phi_{n}(y)
$$


leads to the introduction of the pollutant modes $\phi_{n}(y)$ and their associated spatial decay rates (eigenvalues) $\mu_{n}$ :

$$
\frac{\mathrm{d}}{\mathrm{d} y}\left(h \kappa \frac{\mathrm{d} \phi_{n}}{\mathrm{~d} y}\right)+\left[\mu_{n} u-\lambda\right] h \phi_{n}=0
$$

with

$$
h \kappa \frac{\mathrm{d} \phi_{n}}{\mathrm{~d} y}=0 \text { on } y=a, b .
$$

Modes are only known explicitly for restricted families of exactly solvable test cases, but exist and are countable for any non-negative depth, diffusivity and velocity profiles.

It is conventional to order the modes in increasing values of the spatial decay rates

$$
\mu_{0}<\mu_{1}<\mu_{2}<\ldots
$$

This ordering corresponds to the modes becoming increasingly oscillatory with respect to $y$. Figure 2 shows the first few modes for the illustrative case of a conserved $(\lambda=0)$ solute in a channel with water depth that increases from zero at the left to maximum at the right. Universal properties are: i) the zero mode $\phi_{0}(y)$ is single signed; ii) the $n$ 'th pollutant mode $\phi_{n}(y)$ has $n$ zeros that interlace with the $n+1$ zeros of $\phi_{n+1}(y)$; iii) for $n=1$ there is a position $y_{1}$ such that

$$
\phi_{1}\left(y_{1}\right)=0 \quad \text { with } \quad \phi_{2}(a) \phi_{2}\left(y_{1}\right) \leq 0 \quad \text { and } \quad \phi_{2}(b) \phi_{2}\left(y_{1}\right) \leq 0
$$

In a river with symmetric depth, flow, mixing and decay, $y_{1}$ is at the geometric centre.

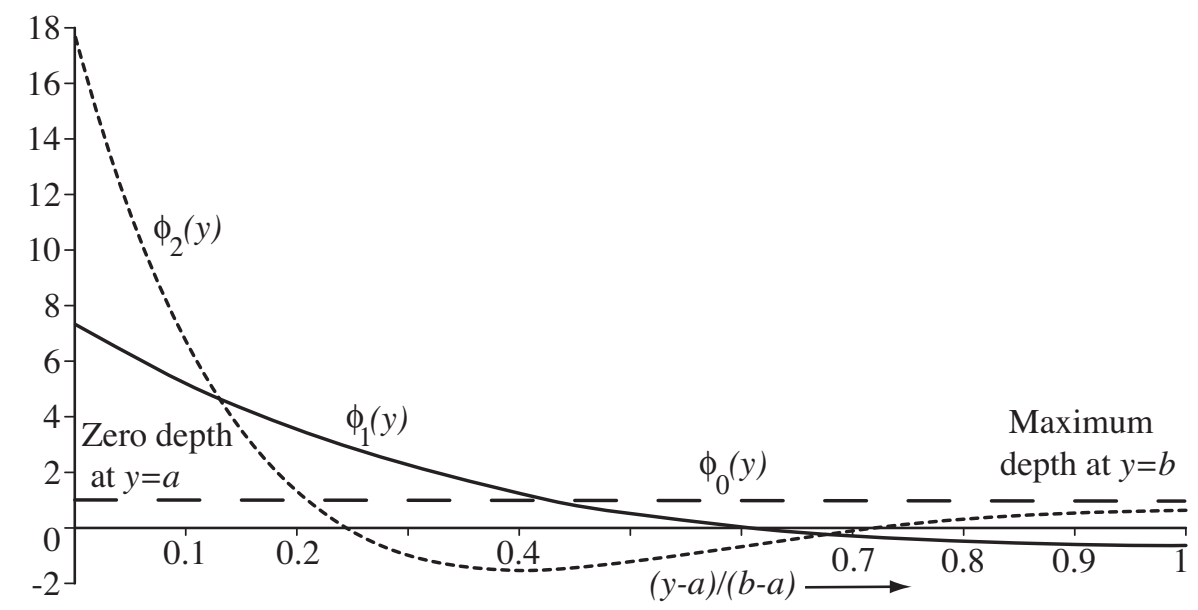

Figure 2: Normalized modes for linearly increasing depth.

If at $x=0$, the cross-river concentration profile is denoted by $f(y)$, or the profile of discharge flux is denoted by $h u f(y)$, then the weights $C_{n}$ can be evaluated from integrals 
across the river

$$
C_{n}=\int_{a}^{b} h u f(y) \phi_{n}(y) d y / \int_{a}^{b} h u \phi_{n}^{2} d y .
$$

Normalizing the modes with respect to the volume flux $Q$ :

$$
\int_{a}^{b} h u \phi_{n}^{2} d y=\int_{a}^{b} h u d y=Q
$$

gives minor simplifications. In particular, for a pollutant flux rate $q$ discharged at the point $y_{p}$, the formula (8) takes the neat form

$$
C_{n}=\frac{q}{Q} \phi_{n}\left(y_{p}\right)
$$

The normalization (9) permits the higher modes to become large in shallow, slow-flowing water, as at the left of figure 2 . The shallow-water concentrations are sensitive to the weights $C_{n}$ and those weights are sensitive to shallow-water positioning of the discharge. For the illustrative case, the shoreline pollution is in excess of five times optimal for the left threetenths in figure 1.

For the solution (3) the lowest mode $\phi_{0}(y)$ is the transverse equilibrium concentration profile and the lowest eigenvalue $\mu_{0}$ is the spatial rate of downstream decay at large distances downstream. If $C_{1}$ is non-zero then the decay-adjusted exponential rate of approach to that transverse equilibrium is $\mu_{1}-\mu_{0}$ with shape $C_{1} \phi_{1}(y)$. The sign of $C_{1}$ determines at which of the two banks there is a decay-adjusted relative pollution excess (as in figure 1). Such shoreline excesses are avoided if $C_{1}=0$. It is clear from equations $(7,10)$ that $C_{1}=0$ can be achieved for a discharge flux confined to the single point $y_{p}=y_{1}$. In that case, the decayadjusted relative exponential rate of approach to the transverse equilibrium is increased to $\mu_{2}-\mu_{0}$ with shape $C_{2} \phi_{2}(y)$ and size $C_{2}=\phi_{2}\left(y_{1}\right) q / Q$. From the inequalities (7), it follows that far downstream at both banks there is a negative departure relative to decay adjusted equilibrium. Shoreline pollution hot-spots (relative to decay adjusted equilibrium) have been eliminated. Consequently, $y_{1}$ is the diffusion centre.

Numerical methods for computing low modes $\phi_{0}(y), \phi_{1}(y)$ of the system $(4,5)$ and for the estimation of the root $y_{1}$, are well established [6]. Instead, this paper pursues exact solutions. One motivation is to sharpen understanding. Another motive is to add decay examples to the stock of non-trivial exact solutions for the benchmark testing of numerical computation schemes for environmental impacts in rivers [10]. 


\section{Flow parameters in straight channels}

Flows in natural streams tend to be turbulent. Elder [3] showed that the local turbulent diffusivities are proportional to the product of the local water depth and longitudinal velocity. This implies $[8,13,14]$, that the longitudinal velocity varies as square-root of the depth and the diffusivity as the three-halves power of the water depth:

$$
u=U\left(\frac{h}{H}\right)^{\frac{1}{2}}, \quad \kappa=K\left(\frac{h}{H}\right)^{\frac{3}{2}} .
$$

The capital letters $H, U$ and $K$ denote the depth, flow and transverse mixing at a reference position in the channel. The depth-linked formulae (11) ensure that in the deepest parts of the channel the velocity is greatest and transverse mixing is most vigorous [2]. It is implicit that the pollutant is sufficiently dilute that it does not significantly modify the turbulence (e.g. applicable to oil in droplets but not to a continuous oil slick).

The illustrative examples in this paper use power-law models for the decay:

$$
\lambda=\Lambda\left(\frac{h}{H}\right)^{\delta+\frac{1}{2}}
$$

For positive (or negative) $\delta$ the ratio $\lambda(y) / u(y)$ increases (or decreases) with depth.

With the representations $(11,12)$ the eigenvalue problem $(4,5)$ becomes

$$
K \frac{d}{d y}\left(\left(\frac{h}{H}\right)^{\frac{5}{2}} \frac{d \phi_{n}}{d y}\right)+\left(\frac{h}{H}\right)^{\frac{3}{2}}\left[\mu_{n} U-\Lambda\left(\frac{h}{H}\right)^{\delta}\right] \phi_{n}=0
$$

with

$$
\left(\frac{h}{H}\right)^{\frac{5}{2}} \frac{d \phi_{n}}{d y}=0 \text { on } y=a, b
$$

For zero decay, the concept of the diffusion centre and the method of modes that is used to identify it, are not restricted to straight non-varying channels [15]. The mathematics looks more daunting because of the need to use of flow-following coordinates [20]. Also, the modes split into downstream evolving $\phi_{n}(x, y)$ and upstream evolving $\hat{\phi}_{n}(x, y)$ adjoints. The diffusion centre is where $\hat{\phi}_{1}\left(x, y_{1}(x)\right)=0$. It is evolving upstream in response to downstream changes to the cross-sectional profile on the length scale $1 / \mu_{2}$ of cross-sectional mixing [15] i.e. it is downstream of the discharge where shoreline pollution excesses might otherwise have occured. With decay, the principal changes would be that the transverse equilibrium $\phi_{0}(x, y)$ ceases to be constant across the channel and has decay $\mu_{0}(x)$ along the flow. 


\section{$5 \quad$ Linearly increasing depth}

For illustrative purposes, the chosen depth profile across the flow is linear increase from zero depth at one side $y=0$ to maximum depth $H$ at the other side $y=B$ :

$$
h(y)=H\left(\frac{y}{B}\right) \text { for } 0<y<B
$$

Decay proportional to the flow speed $\lambda=\Lambda(h / H)^{\frac{1}{2}}$ (i.e. $\delta=0$ ) has no effect on the modes. The modes are unchanged from those for a conserved solute. The lowest mode is constant across the channel $\phi_{0}=1$. Higher modes (normalized) can be represented [15]:

$$
\phi_{n}=\frac{\left(a_{n}(y / B)^{\frac{1}{2}}\right)^{-1} \sin \left(a_{n}(y / B)^{\frac{1}{2}}\right)-\cos \left(a_{n}(y / B)^{\frac{1}{2}}\right)}{(y / B)\left(\frac{5}{2}\right)^{\frac{1}{2}}\left\{1+\frac{\cos \left(a_{n}\right) \sin \left(a_{n}\right)}{a_{n}}-2 \frac{\sin \left(a_{n}\right)^{2}}{a_{n}^{2}}\right\}^{\frac{1}{2}}} .
$$

The square-root terms in the denominator that normalize the modes for arbitrary $a_{n}$.

The no-flux boundary condition (14) at $y=B$ restricts the $a_{n}$ to being non-negative roots of the equation:

$$
\sin \left(a_{n}\right)=-\frac{3 a_{n} \cos \left(a_{n}\right)}{a_{n}^{2}-3}, a_{1}=5.763, a_{2}=9.095, a_{n} \approx n \pi-\frac{3}{n \pi} .
$$

The construction of figure 1 from the modal solution (3) used up to 40 modes to achieve high accuracy. Figure 2 shows the $n=0,1,2$ normalized modes. The diffusion centre is at

$$
y_{1} / B=0.608
$$

Decay proportional to the flow speed, augments the eigenvalues $\mu_{n}$ by $\Lambda / U$ :

$$
\mu_{n}=\frac{K a_{n}^{2}}{4 U B^{2}}+\frac{\Lambda}{U}
$$

For $n=2$, transverse mixing and decay give equal contributions to $\mu_{n}$ for $\Lambda B^{2} / K \approx 20$. For larger rates of decay shoreline pollution becomes insignificant unless the discharge is irresponsibly close to shore. The subsequent computations are restricted to $\Lambda B^{2} / K \leq 10$.

In the context of pollution minimisation from sudden discharges of pollutants, Daish [2] used piecewise linear depth profiles to construct a wide range of test problems. The following three sub-sections concern the linear depth profile (15), but with decay profiles corresponding to uniform consumption by bacteria, to evaporation and to break up by turbulence. The diffusion centre $y_{1}$ is found to deviate by $\pm B / 8$ from the non-decaying reference case (18). 


\subsection{Constant decay}

Radioactive decay or consumption by bacteria (at a rate unaffected by sunlight or turbidity) are examples of constant temporal decay $\lambda=\Lambda$ (i.e. $\delta=-\frac{1}{2}$ ) .

The exact solutions for the pollutant modes (not normalised) have an explicit solution proportional to a Whittaker function with first and third arguments imaginary:

$$
\phi_{n}=(-1)^{n} \frac{\text { Whittaker } M\left(\mathrm{i} \Lambda /\left(\mu_{n} U\right), \frac{3}{2}, 4 \mathrm{i}\left(\mu_{n} U B y / K\right)^{\frac{1}{2}}\right)}{(y / B)} .
$$

The sign switching $(-1)^{n}$ is included so that, for compatibility with figure $2, \phi_{1}(y)$ exhibits a change from positive for small $y$ to negative for large $y$. The no-flux boundary condition (14) at $y=B$ yields an equation satisfied by the eigenvalues $\mu_{n}$ :

$$
\begin{aligned}
& \left(2+\frac{\mathrm{i} \Lambda B}{\left(\mu_{n} U K\right)^{\frac{1}{2}}}\right)\left[\text { Whittaker } M\left(\mathrm{i} \Lambda B /\left(\mu_{n} U K\right)^{\frac{1}{2}}, \frac{3}{2}, 4 \mathrm{i}\left(\mu_{n} U B^{2} / K\right)^{\frac{1}{2}}\right)\right. \\
& \text { - Whittaker } \left.M\left(\mathrm{i} \Lambda /\left(\mu_{n} U\right)+1, \frac{3}{2}, 4 \mathrm{i}\left(\mu_{n} U B^{2} / K\right)^{\frac{1}{2}}\right)\right] \\
& =2 \mathrm{i}\left(\mu_{n} U B^{2} / K\right)^{\frac{1}{2}} \text { Whittaker } M\left(\mathrm{i} \Lambda /\left(\mu_{n} U\right), \frac{3}{2}, 4 \mathrm{i}\left(\mu_{n} U B^{2} / K\right)^{\frac{1}{2}}\right) \text {. }
\end{aligned}
$$

Once an eigenvalue $\mu_{n}$ is evaluated, the shape of the mode $\phi_{n}(y)$ is given by equation (20). Figure 3 shows the normalized first mode shapes for three values of the decay parameter. For zero decay the first mode $\phi_{1}(y)$ and discharge centre $y_{1}$ are identical with those in figure 2 and equation (18). The effects of decay on $\phi_{1}(y)$ are most marked near the beach. For a fixed distance downstream, the slower flow in shallow water gives more time for the contaminant to be depleted.

\begin{tabular}{|c|c|}
\hline$\Lambda B^{2} / K$ & $y_{1} / B$ \\
\hline 0 & 0.608 \\
2 & 0.637 \\
4 & 0.657 \\
6 & 0.673 \\
8 & 0.685 \\
10 & 0.696 \\
\hline
\end{tabular}

Table 1: Optimal discharge positions for linearly increasing depth and constant decay.

From a $\phi_{1}(y)$ curve it is straightforward to compute the root $y_{1}$. Table 1 lists $\Lambda B^{2} / K$, $y_{1} / B$ pairings including those appropriate to the curves shown in figure 3 . Constant decay shifts the diffusion centre towards the deeper water by an amount proportional to $\Lambda$. 


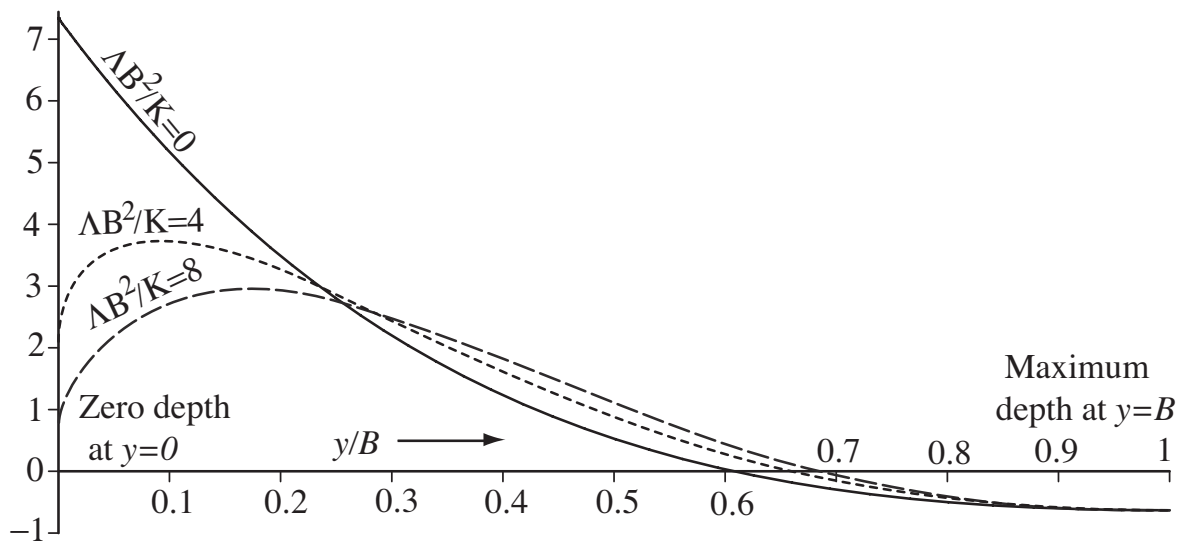

Figure 3: First mode $\phi_{1}(y)$ for constant decay $\Lambda$ and linearly increasing depth.

\subsection{Decay that decreases with depth}

The decay can decrease with depth if $c(y, t)$ is a number count of bacteria which are killed by sunlight [7] only in the top few metres of the water column. A second example is removal at the bed (feeding by marine micro-organisms, fungi or yeasts). A third example is air-water contaminant exchange at the surface. For example, evaporation accounts for largest loss in oil volume during the early stages $[11,12]$. Fingas $[4,5]$ notes that light crude oils can lose as much as 75 per cent of their original volume within the first few days after a spill; mediumweight crudes might lose as much as 40 per cent of the original volume. Heavy crude or residual oils, on the hand, will probably only lose about 10 per cent of their volume in the first few days.

An exactly solvable model problem that illustrates decay decreasing with depth is $\lambda=$

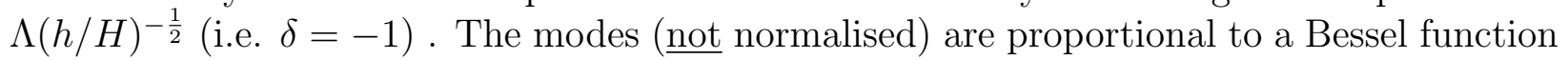
of non-integer-order:

$$
\phi_{n}=\frac{\operatorname{Bessel} J\left(\left(\frac{9}{4}+4 \Lambda B^{2} / K\right)^{\frac{1}{2}}, 2\left(\mu_{n} U B y / K\right)^{\frac{1}{2}}\right)}{(y / B)^{\frac{3}{4}}} .
$$

The no-flux boundary condition (14) at $y=B$ yields the eigenvalue equation with roots $\mu_{n}$ :

$$
\begin{aligned}
& \left(\frac{9}{4}+4 \Lambda B^{2} / K\right)^{\frac{1}{2}} \text { Bessel } J\left(\left(\frac{9}{4}+4 \Lambda B^{2} / K\right)^{\frac{1}{2}}, 2\left(\mu_{n} U B y / K\right)^{\frac{1}{2}}\right) \\
& -2\left(\mu_{n} U B^{2} / K\right)^{\frac{1}{2}} B \text { essel } J\left(\left(\frac{9}{4}+4 \Lambda B^{2} / K\right)^{\frac{1}{2}}+1,2\left(\mu_{n} U B^{2} / K\right)^{\frac{1}{2}}\right) \\
& =\frac{3}{2} \text { Bessel } J\left(\left(\frac{9}{4}+4 \Lambda B^{2} / K\right)^{\frac{1}{2}}, 2\left(\mu_{n} U B^{2} / K\right)^{\frac{1}{2}}\right) .
\end{aligned}
$$




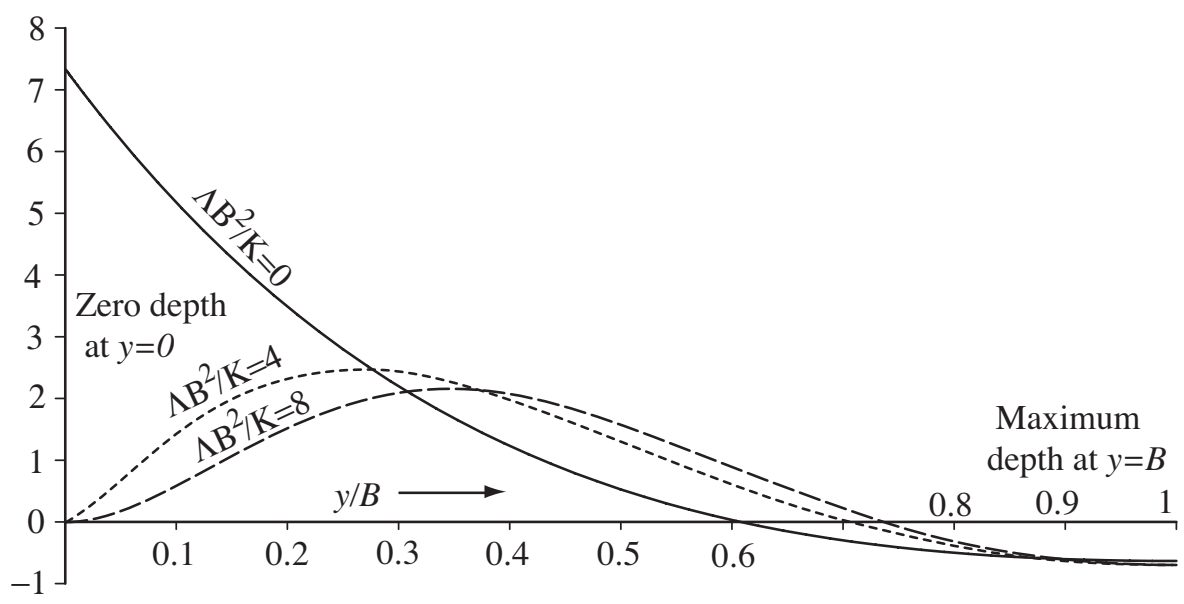

Figure 4: First mode $\phi_{1}(y)$ for decay that decreases with depth.

\begin{tabular}{|c|c|}
\hline$\Lambda B^{2} / K$ & $y_{1} / B$ \\
\hline 0 & 0.608 \\
2 & 0.676 \\
4 & 0.705 \\
6 & 0.723 \\
8 & 0.736 \\
10 & 0.747 \\
\hline
\end{tabular}

Table 2: Discharge positions for decay that decreases with depth.

Figure 4 and table 2 respectively show the effects of the strength of the decay on the $\phi_{1}$ pollutant mode and upon the diffusion centre. Again, for zero decay the first mode $\phi_{1}(y)$ and discharge centre $y_{1}$ are identical with those in figure 2 and equation (18). For non-zero decay, the singularity in $\lambda(y)$ at the beach $y=0$, gives a jump to zero for $\phi_{1}(0)$. Also, the displacement of the optimal discharge site towards the deeper part of the channel scales as the square-root of $\Lambda$.

\subsection{Decay that increases with depth more than the velocity}

The dissolution of oils or break up of clay flocs is most rapid in regions of the flow where the turbulence is energetic. In this sub-section, the decay is modelled $\lambda=\Lambda(h / H)^{\frac{3}{2}}$, as proportional to the turbulent mixing (i.e. $\delta=1$ ) and increasing with depth more strongly than the velocity (11).

The pollutant modes (not normalised) are proportional to Whittaker function modes with 
all three arguments real:

$$
\phi_{n}=\frac{\text { Whittaker } M\left(\frac{1}{2} \mu_{n} U B /(\Lambda K)^{\frac{1}{2}}, \frac{3}{4}, 2(\Lambda / K)^{\frac{1}{2}} y\right)}{(y / B)^{\frac{5}{4}}} .
$$

The no-flux boundary condition at $y=B$ yields an equation satisfied by the eigenvalues $\mu_{n}$ :

$$
\begin{aligned}
&\left(\frac{5}{4}+\frac{\mu_{n} U B}{2(\Lambda K)^{\frac{1}{2}}}\right) {\left[\text { Whittaker } M\left(\frac{1}{2} \mu_{n} U B /(\Lambda K)^{\frac{1}{2}}, \frac{3}{4}, 2(\Lambda / K)^{\frac{1}{2}} B\right)\right.} \\
&\left.- \text { Whittaker } M\left(\frac{1}{2} \mu_{n} U B /(\Lambda K)^{\frac{1}{2}}+1, \frac{3}{4}, 2(\Lambda / K)^{\frac{1}{2}} B\right)\right] \\
&=(\Lambda / K)^{\frac{1}{2}} B \text { Whittaker } M\left(\frac{1}{2} \mu_{n} U B /(\Lambda K)^{\frac{1}{2}}, \frac{3}{4}, 2(\Lambda / K)^{\frac{1}{2}} B\right) .
\end{aligned}
$$

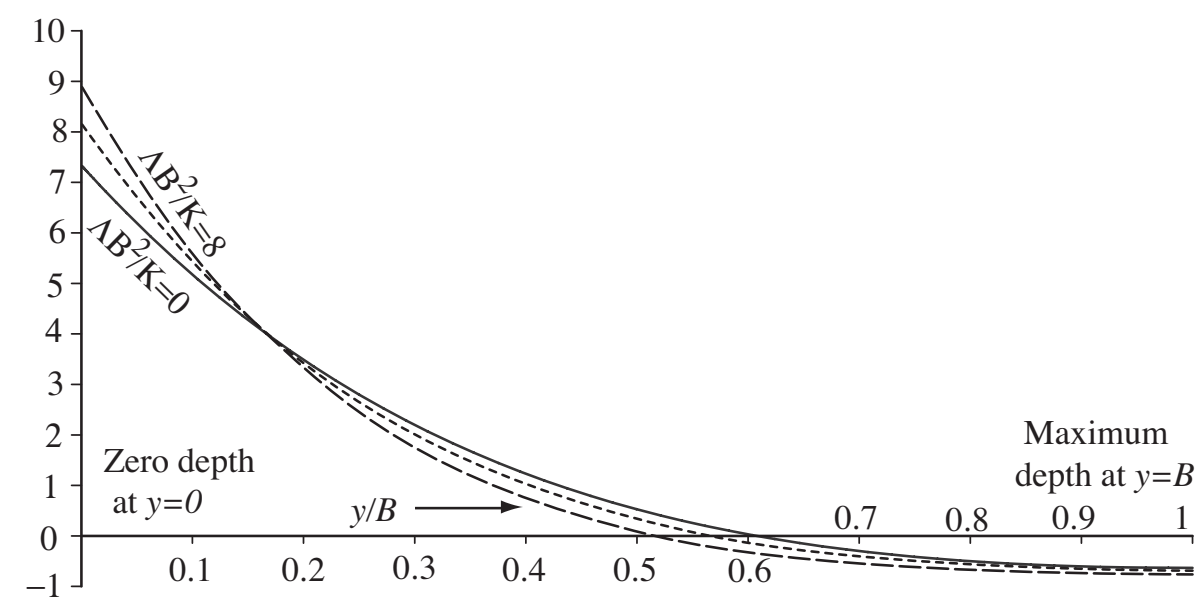

Figure 5: First mode $\phi_{1}(y)$ for decay that increases with depth.

\begin{tabular}{|c|c|}
\hline$\Lambda B^{2} / K$ & $y_{1} / B$ \\
\hline 0 & 0.608 \\
2 & 0.589 \\
4 & 0.568 \\
6 & 0.544 \\
8 & 0.518 \\
10 & 0.489 \\
\hline
\end{tabular}

Table 3: Discharge positions for decay that increases with depth.

Figure 5 and table 3 respectively show the first pollutant mode $\phi_{1}(y)$ and the optimum discharge site $y_{1}$ for a few values of $\Lambda B^{2} / K$. Once more, for zero decay the first mode $\phi_{1}(y)$ and discharge centre $y_{1}$ are identical with those in figure 2 and equation (18). Contrary to the previous two sub-sections, the diffusion centre shifts to the shallower water. 


\section{Ratio $\lambda / u$ as an indicator for changes in $y_{1}$}

A physical interpretation of the ratio $\lambda(y) / u(y)$ is as the local spatial decay rate for the contaminant at the cross-channel location $y$. For the special case of linear depth, it was observed in $\S 5$ that if $\lambda(y) / u(y)$ is constant (i.e. $\delta=0)$, then the modal shapes $\phi_{n}(y)$ are not effected by decay and the spatial decay rates $\mu_{n}$ for the modes are merely augmented by that constant spatial decay rate $\lambda / u$. It is evident from the square-bracketed terms in equation (4), that the same is true whatever the depth profile $h(y)$.

It is natural to investigate whether the depth-dependence of the ratio $\lambda(y) / u(y)$, or the sign and magnitude of the exponent $\delta$ in the power-law model (12), can be used as an indicator for changes to the position of the diffusion centre. In $\S 5.1$ and figure $(3), \lambda(y) / u(y)$ decreases with negative exponent $\delta=-\frac{1}{2}$ and the diffusion centre shifts weakly to deeper water. In $\$ 5.2$ and figure $(4), \lambda(y) / u(y)$ decreases more strongly with negative exponent $\delta=-1$ and the diffusion centre shifts more strongly to deeper water. Finally, in $\S 5.3$ and figure (5), $\lambda(y) / u(y)$ increases with positive exponent $\delta=1$ and the diffusion centre shifts to shallower water. Hence, the diffusion centre is shifted to deeper or to shallower water accordingly as the ratio $\lambda(y) / u(y)$ decreases or increases with water depth (i.e. $\delta$ negative or positive).

\section{Concluding remarks}

Avoidable shoreline pollution excesses can be large for steady discharges close to shore. The special cases solved in this paper sharpen insight about how loss mechanisms displace the best siting of steady discharges away from the site appropriate to non-decaying solutes. The mixing or diffusion centre is shifted to deeper or to shallower water accordingly as the spatial decay rate (i.e. ratio $\lambda(y) / u(y))$ decreases or increases with water depth.

The three families of exact modes $(20,22,24)$ together with the exact series solution (3) and weights (8) permit the construction of exact solutions that include decay. Such exact solutions extend the scope for for the benchmark testing [10] of numerical computation schemes for environmental impacts in rivers.

Commonly, a steady discharge will comprise a mixture of pollutants with different decay processes and rates. The chosen discharge site will be a compromise between the diffusion centre for the constituent pollutants. For the wide range of cases considered in this paper, the optimal discharge position for the different decay processes is never found to be more than one eighth of a channel breadth from the site appropriate to non-decaying solutes. For each decaying constituent the displacement between the discharge site and the diffusion centre for that constituent, corresponds to shoreline pollution excesses less than a factor of 0.4 above optimal (as contrasted to the factor of 4.0 excess at which figure 1 is truncated). 
The variety of exact solutions in this paper illustrate the robustness of the commonsense policy that, to avoid large shoreline pollution excesses from any of the constituents in a mixture of pollutants, the discharge should be sited more or less in the middle of the river.

\section{Acknowledgements}

We thank the referees for constructive comments. The first author would like to thank Bayelsa State Government of Nigeria for financial support through the award of a $\mathrm{PhD}$ research fellowship.

\section{References}

1. Bikangaga, J. H. and Nassehi, V.: 1995, Application of Computer Modelling Techinques to the Determination of Optimum Efluent Discharge Policies in Tidal Water Systems. Wat. Res.,29,10, 2367-2375.

2. Daish, N. C.: 1985, The Optimal discharge profiles for sudden contaminant releases in steady, unform open-channel flow. J. Fluid Mech., 159, 303-321.

3. Elder, J. W.: 1959, The dispersion of marked fluid in turbulent shear flow. J. Fluid Mech., 5, 544-560.

4. Fingas, M.: 1994a, Studies on the evaporation of oil spills. Seventeenth Arctic Marine Oil Spill Program (AMOP) Tecnical Seminar, Vancouver, Canada, June 8-10, 1994, Vancouver, British Columbia: Environment Canada, 189-210.

5. Fingas, M.: 1985, A literature review of the physics and predictive modelling of oil spill evaporation. Journal of Hazardous Materials, 42,157-175.

6. Fox, L.: 1957, The numerical solution of two-point boundary value problems, Oxford University Press, London UK.

7. Gould, D.J. and Munro, D.: 1981, Relevance of Microbial Mortality to outfall Design. Coastal Discharges, Thomas Telford, London, U.K., 45-50.

8. Kay, A.: 1987, The Effect of Cross-Stream Depth Variations upon Contaminant Dispersion in a Vertically Well-mixed Current. Estuarine, Coastal and Shelf Science, 24, $177-204$.

9. Nassehi, V. and Bikangaga, J. H.: 1993, A mathematical model for the hydrodynamics and pollutants transport in long and narrow tidal rivers. Appl. Math. Modelling, 17, 415-422. 
10. Nassehi, V. and Passone, S.: 2005, Testing Accuracy of Finite Element and Random Walk Schemes in Prediction of Pollutant Dispersion in Coastal Waters. Environmental Fluid Mech., to appear.

11. Overstreet, R. and Galt, J.A.: 1995, Physical Processes Affecting the Movement and Spresding of Oils in Inland Waters. NOAA/Hazardous Materials Response and Asssessment Division Seattle, Washingto, HAZMAT Report 95-7.

12. Reddy, G.S. and Brunet, M.: 2003, Numerical Prediction of Oil Slick Movement in Gabes Estuary, www.fluidyn.com/Research.

13. Rutherford, J. C.: 1994, River Mixing, Wiley, Chichester UK.

14. Smith, R.: 1981, Effect of non-uniform currents and depths variations upon steady discharges in shallow water. J. Fluid Mech., 110, 373-380.

15. Smith, R.: 1982, Where to put a steady discharge in a river. J. Fluid Mech., 115, 1-11.

16. Smith, R.: 1996, Horizontal fractionation of rising and sinking particles in wind-affected currents. J. FLuid Mech.., 316, 325-334.

17. Smith, R.: 2004, Mixing Center of a Channel. J. Hydraulic Engineering, 130, 2, 165-169.

18. Yoo, M. K., Cho, S. W., and Jun, K. S.: 2003, Unsteady Dispersion of Nonconservative Pollutants in Natural Rivers. www.kfki.baw.de/conferences.

19. Yotsukura, N. and Cobb, E. D.: 1972, Transverse diffusion of solutions in natural streams. U. S. Geo. Survey Paper, no.582 C.

20. Yotsukura, N. and Sayre, W.W.: 1976, Transverse mixing in natural channels. U. $S$. Geo. Survey Paper, no.582 C. 